\title{
Evidence of spin disorder at the surface-core interface of oxygen passivated Fe nanoparticles
}

\author{
L. Del Bianco, ${ }^{\text {a) }}$ A. Hernando, M. Multigner, and C. Prados \\ Instituto de Magnetismo Aplicado, UCM-RENFE, P.O. Box 155, Las Rozas, Madrid 28230, Spain
}

J. C. Sánchez-López and A. Fernández

Departamento de Química Inorgánica, Instituto de Ciencia de Materiales de Sevilla, Centro de Investigaciones Cientificas Isla de la Cartuja, Avda. A. Vespuccio, s/n. Sevilla 41092, Spain

C. F. Conde and A. Conde

Departamento de Física de la Materia Condensada, Universidad de Sevilla-Instituto de Ciencia de Materiales de Sevilla, P.O. Box 1065, Sevilla 41080, Spain

(Received 10 February 1998; accepted for publication 1 May 1998)

\begin{abstract}
Hysteresis, thermal dependence of magnetization, and coercivity of oxide coated ultrafine $\mathrm{Fe}$ particles prepared by inert gas condensation and oxygen passivation have been studied in the 5-300 $\mathrm{K}$ range. The results are found to be consistent with a spin-glasslike state of the oxide layer inducing, through exchange interaction with the ferromagnetic core, a shift of the field cooled hysteresis loops at temperatures below the freezing at approximately $50 \mathrm{~K}$. (C) 1998 American Institute of Physics. [S0021-8979(98)07315-0]
\end{abstract}

\section{INTRODUCTION}

The study of the magnetic properties of fine particles has been traditionally urged by both a technological and theoretical interest connected with the possibility of developing a better understanding of magnetic phenomena related to size effects. In recent times, new methods of synthesis (inert gas condensation, layer deposition, ultrarapid quenching, mechanical attrition, aerosol) have been used to fabricate magnetic systems with characteristic dimensions on a nanometer scale. Innovative nanostructured systems with excellent soft or hard magnetic properties ${ }^{1,2}$ or with giant magnetoresistance response $\mathrm{s}^{3}$ have been created, which at present play an important role in modern technology and research.

That was also the occasion for a renewed interest in the field of magnetism of ultrafine particles, probably in view of their potential applications in high density magnetic recording or as ferrofluids, which has invariably led to the discovery of unexpected magnetic behavior strictly depending on the reduced dimensional regime. ${ }^{4}$

A number of research works has been published during the last few years dealing with the magnetic behavior of nanometric $\mathrm{Fe}, \mathrm{Co}$, and $\mathrm{Ni}$ nanocrystalline particles, mainly prepared by the inert gas condensation method, and presenting different degrees of surface oxidation. ${ }^{5-9}$ In this article, once more attention has been focused on the low temperature magnetic behavior of oxide coated ultrafine Fe particles and a different explanation, based on the spin-glasslike state of the oxide layer, is proposed to account for the observed results.

\footnotetext{
a) Author to whom correspondence should be addressed; on leave from: Dipartimento di Fisica, Università di Bologna and INFM, Viale Berti Pichat 6/2, 40126 Bologna, Italy.
}

\section{EXPERIMENT}

Nanocrystalline Fe particles have been prepared by evaporation of iron in a tungsten boat at $1773 \mathrm{~K}$. The pressure of gas phase during evaporation was $133 \mathrm{~Pa}$ (1 Torr) helium.

Through interatomic collision with the inert gas atoms, the evaporated atoms lose kinetic energy and condense as an ultrafine powder that accumulates on a cold finger. ${ }^{10}$ The passivation was achieved by dosing oxygen (266 $\mathrm{Pa}$ for 10 min) before opening the chamber to air. The loose powder was smoothly stripped off and stored in air.

Figure 1(a) shows a transmission electron microscope (TEM) micrograph of the passivated ultrafine powder, which has been carried out through a Philips CM200 microscope working at $200 \mathrm{kV}$. For the TEM examination the powder was previously dispersed in ethanol by sonication and dropped on a conventional carbon coated copper grid. The material consists of nanometer sized particles nearly spherical (15-40 nm in diameter) presenting a core-shell structure. The dark inner core, visible in the micrograph, corresponds to metallic iron, whereas the surrounding light layer is expected to be the oxide phase.

$\mathrm{X}$-ray diffraction (XRD) analysis of the as-prepared ultrafine powder [Fig. 1(b)] reveals the presence of pure $\alpha$-Fe and some small and very broad features at the position of the peaks for $\gamma-\mathrm{Fe}_{2} \mathrm{O}_{3}$ and $\mathrm{Fe}_{3} \mathrm{O}_{4}$ (it is not possible to differentiate between these two phases by XRD as their lattice parameters are very similar). These small peaks can be due to a poor crystallization of these compounds in the thin passivation coating. The presence of oxide species at the surface of the passivated nanocrystalline powders was also fully confirmed by x-ray photoelectron spectroscopy. ${ }^{11}$

The magnetic behavior of the ultrafine powder has been investigated through a commercial semiconducting quantum interference device (SQUID) magnetometer supplying a 


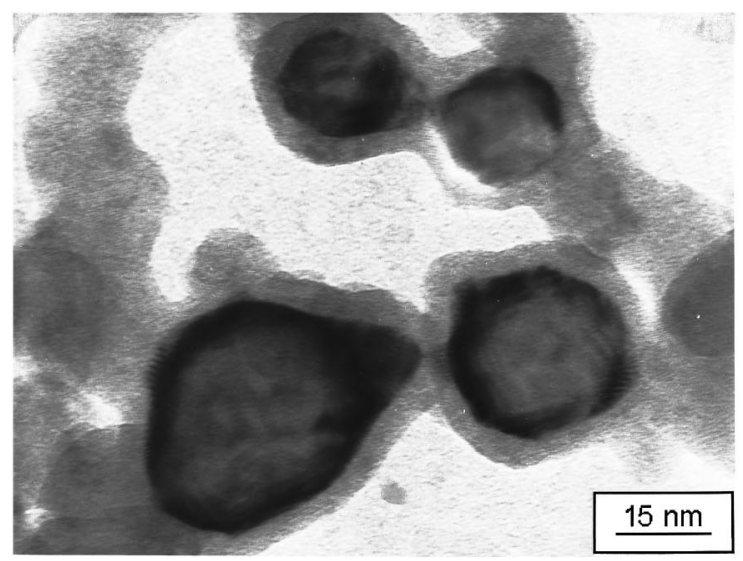

(a)

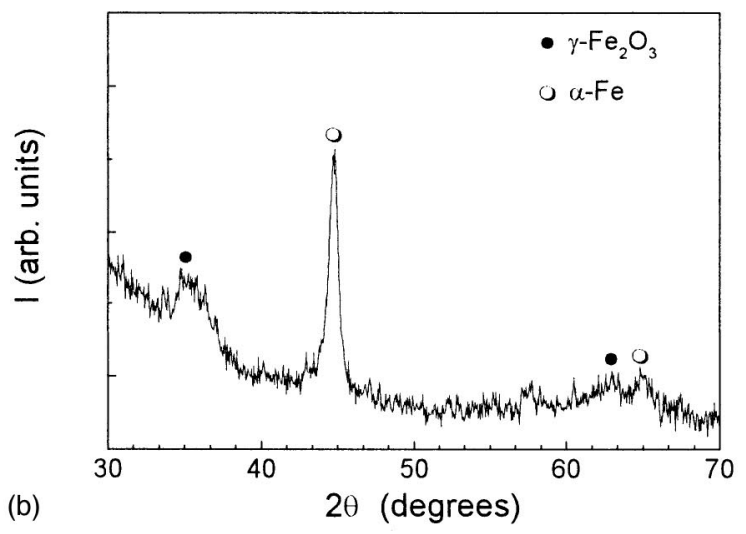

FIG. 1. TEM micrograph (a) and x-ray diffraction spectrum (b) of the oxygen passivated ultrafine Fe powder.

magnetic field up to $55 \mathrm{kOe}$ and operating in the $5-300 \mathrm{~K}$ temperature range.

\section{RESULTS AND DISCUSSION}

In Fig. 2, the temperature dependence of the magnetization, measured at an applied field $H=50 \mathrm{kOe}$ is shown. Full square curves have been measured after zero-field cooling and open circles corresponds to the field cooling (at $50 \mathrm{kOe}$ )

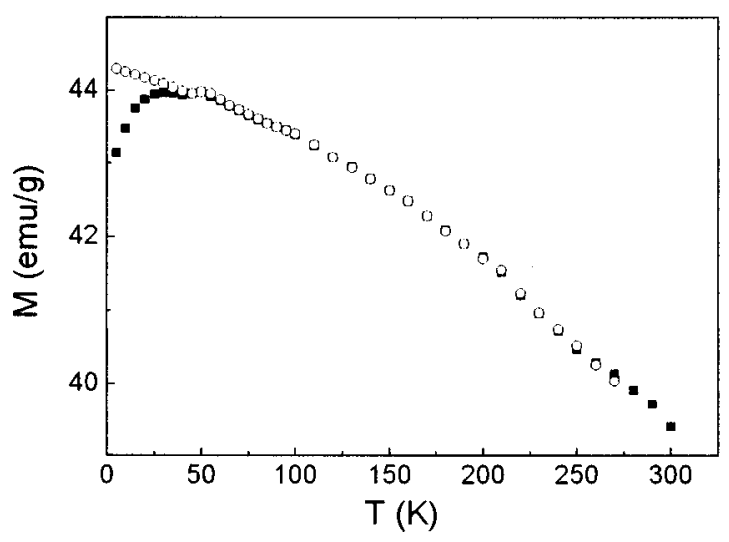

FIG. 2. Zero-field cooled (full squares) and field cooled (open circles) temperature dependence of magnetization for the ultrafine Fe powder (applied field $H=50 \mathrm{kOe}$ ).

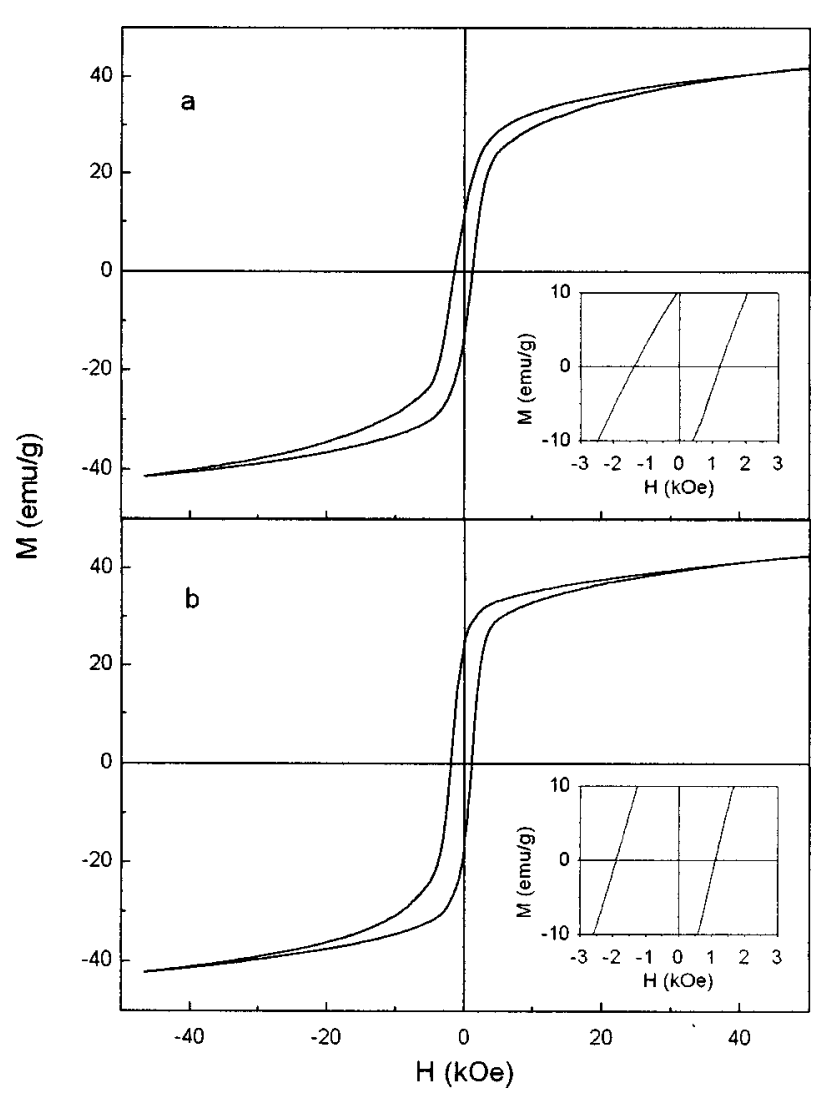

FIG. 3. Hysteresis loops measured at $T_{m}=5 \mathrm{~K}$ after cooling in zero field (a) and after cooling from room temperature in a field $H=20 \mathrm{kOe}$ (b). In the inset: central part of the loop.

procedure. Up to approximately $30-50 \mathrm{~K}$, the magnetization in the zero-field cooling curve is seen to rise and then decreases regularly up to room temperature.

In Figs. 3(a) and 3(b), hysteresis loops measured at a temperature of $5 \mathrm{~K}$ according to the conventional zero-field cooled (ZFC) and field cooled (FC) methods are displayed. The field applied at $300 \mathrm{~K}$ for the field cooling procedure was of $20 \mathrm{kOe}$. It has been observed that the ZFC loop is symmetric about the origin, whereas after field cooling, a shift of the loop is experienced. The measurements have been repeated at different measuring temperatures $T_{m}$, following the same procedure. In Fig. 4, the coercivity $\left(H_{c}\right)$ of the FC loops (curve a) and the shift (curve b) between ZFC and FC loops is reported as a function of $T_{m}$. It is noteworthy that the loop shift decreases with increasing $T_{m}$ and vanishes near $50 \mathrm{~K}$, namely where the maximum in the magnetization against the temperature curve is observed (Fig. 2). The coercivity also decreases abruptly with temperature going from about 1500 Oe at $5 \mathrm{~K}$ to 200 Oe at room temperature and following a temperature dependence similar to the loop shift.

It is well known that a shift of a FC hysteresis loop may be originated by an exchange anisotropy effect determined by an exchange coupling between a ferromagnetic and an antiferromagnetic (or ferrimagnetic) phase in intimate contact so that the exchange interaction can propagate through the interface. ${ }^{12} \mathrm{~A}$ necessary requirement for the establishment of exchange anisotropy is that the field cooling is per- 


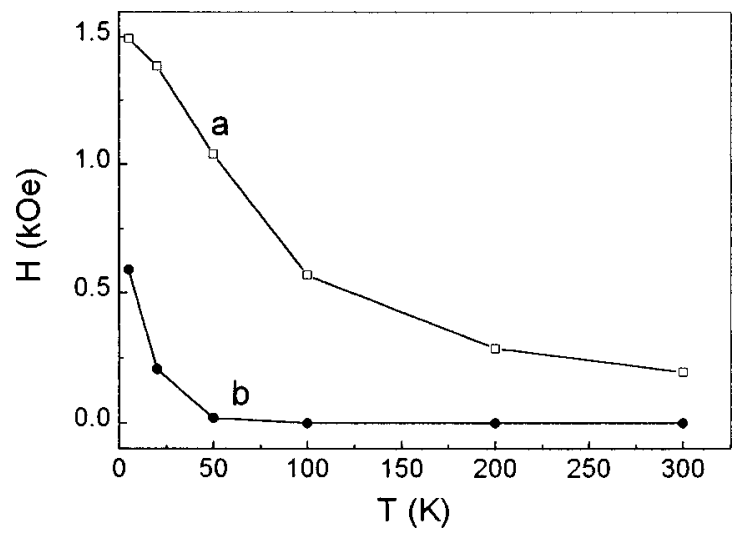

FIG. 4. Coercivity (curve a) and shift (curve b) of the hysteresis loops, measured after cooling in a field $H=20 \mathrm{kOe}$, as a function of the measuring temperature.

formed across the Néel temperature of the antiferromagnetic.

Owing to the particular structural configuration of the $\mathrm{Fe}$ particles, an exchange anisotropy effect was expected. However, the magnetic order-disorder transition temperatures of $\gamma-\mathrm{Fe}_{2} \mathrm{O}_{3}(\sim 1020 \mathrm{~K})$ and $\mathrm{Fe}_{3} \mathrm{O}_{4}(858 \mathrm{~K})$, namely, the $\mathrm{Fe}$ oxides identified by XRD, and also of $\mathrm{FeO}(198 \mathrm{~K})$ are considerably higher than the temperature above which the loop shift disappears.

On the other hand, further evidence of the only necessity, in order to observe the shift, to go through the critical temperature of $50 \mathrm{~K}$ during the field cooling procedure, has been obtained by performing a FC loop at $5 \mathrm{~K}$, taking care to apply the field at $60 \mathrm{~K}$. In this case too, a shift of the FC loop of the same entity as in Fig. 3(b) has been induced.

We propose that the shift of the hysteresis loop is determined by an exchange interaction between the ferromagnetic core of the ultrafine particles and the oxide surface with spinglasslike magnetic behavior and freezing temperature $T_{f}$ $=50 \mathrm{~K}$. In this hypothesis, the application of the field at $T$ higher than $T_{f}$ forces the magnetization of the particle core to lie along the field direction. Due to the exchange coupling with the core spins, the spins at the oxide layer assume a preferential orientation which becomes frozen below $T_{f}$. In turn, below $T_{f}$, the presence of the surface frozen spins favors the ferromagnetic core of the particle being magnetized in the field cooling direction, resulting in a shift of the hysteresis loops. ${ }^{13}$

One characteristic of a spin glass is to possess multiple stable configurations of the frozen state. The application of a strong field, at a temperature lower than freezing, can force the spins to align along the field direction. As a matter of fact, the increase of the magnetization with temperature up to about $50 \mathrm{~K}$ (Fig. 2) can be accounted for by invoking thermally activated transitions toward the spin configuration (favored by the presence of the magnetic field) in the oxide coating of the Fe particles.

Accordingly, in order to support the hypothesis of a spin-glasslike character of the oxide layer, an hysteresis loop has been measured at $5 \mathrm{~K}$, after field cooling from $20 \mathrm{~K}$. In fact, for the above considerations, it was expected that the field cooling procedure, even if carried out starting from a

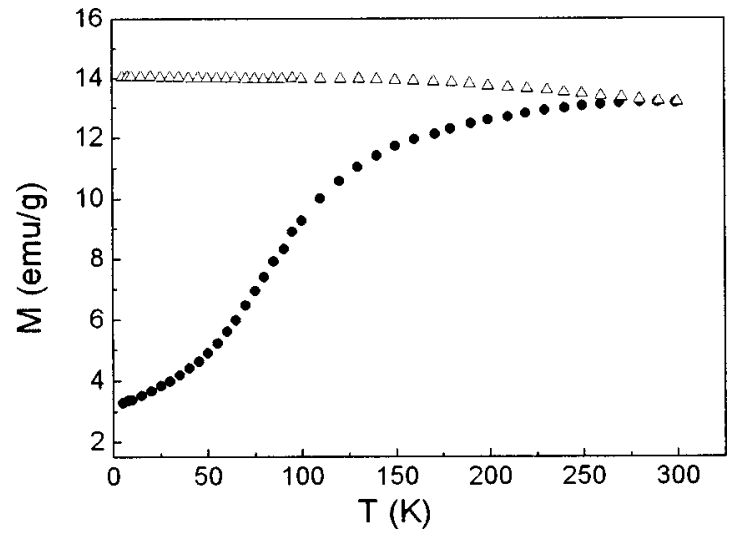

FIG. 5. Zero-field cooled (circles) and field cooled (triangles) magnetization as a function of temperature at an applied field $H=800$ Oe.

temperature lower than $T_{f}$, could select a spin configuration and lead therefore to a displacement of the loop at $5 \mathrm{~K}$. A minor (with respect to the case of field cooling from $300 \mathrm{~K}$ ) but considerable shift of about 150 Oe has been indeed observed.

With regard to the temperature dependence of the coercivity [Fig. 4(a)], a similar trend (even if relative to ZFC loops) for Fe particles prepared in the same way, was already observed by Gangopadhyay et al. ${ }^{14}$ and explained through a "shell-core" model based on the presence, at the particle surface, of an oxide coating in the form of very small crystallites, superparamagnetic above a blocking temperature of $30-40 \mathrm{~K}$.

However, the high coercivity value at $5 \mathrm{~K}$ and the strong decrease with increasing temperature [Fig. 4(a)] may be equally well accounted for considering that the presence of the frozen surface constitutes a strong hindrance to the reversal of the ferromagnetic phase. Moreover, it must be considered that, owing to the small size of the Fe core, some particles may be superparamagnetic at room temperature, which would result in a further decrease of $H_{c}$. This last hypothesis has been indeed confirmed by performing an usual ZFC-FC magnetization against temperature measurement at an applied field $H=800 \mathrm{Oe}$ (Fig. 5). Note that this weak field, in comparison with that used in Fig. 2, allows us to obtain information about the possible superparamagnetic behavior of the Fe cores, whereas the result of Fig. 2 can be correlated with the magnetic behavior of the oxide coating. It is noteworthy that the ZFC magnetization in Fig. 4 does not show a defined maximum and that the $\mathrm{ZFC}$ and $\mathrm{FC}$ branches remain separated up to practically room temperature. This behavior is compatible with a wide size distribution of the particle cores which, therefore, enter the superparamagnetic regime at different temperatures.

Mössbauer spectroscopy studies by Haneda and Morrish ${ }^{15}$ and by Linderoth et al. ${ }^{16}$ on surface oxidized $\mathrm{Fe}$ nanoparticles revealed that the surface layer consisted of very small crystallites with spinel structure and that a large spin canting characterized the oxide phase.

A canting of the spins was also observed by Kodama et al. ${ }^{17}$ at the surface of ferrite nanoparticles. In that case too, it was found that the presence of the canted spins, arising 
from reduced coordination and broken exchange bonds between surface spins, was compatible with a spin-glasslike behaviour of the surface itself. It should be remarked that the behavior of the surface becomes relevant due to the very reduced dimensions of the particles so that the number of atoms at the surface is comparable to the number of atoms at the core. Recently, computer modeling of the influence of surface disorder and spin uncompensation on the magnetic properties of nanoparticles and on the exchange anisotropy of thin films has been reported. ${ }^{18}$

In our case, assuming that the oxide layer is made up of small crystallites, it follows that a high percentage of the total number of spins of the oxide is located at the crystallites surface, more properly indicated as the interfacial or grain boundary region, ${ }^{19}$ as well as at the oxide-Fe interface which would therefore be (in our opinion) the ultimate responsibility of the observed exchange anisotropy effect.

\section{CONCLUSION}

In conclusion, an approach has been proposed in order to explain the low temperature magnetic behavior (temperature dependence of magnetization and coercivity) of oxide coated ultrafine Fe particles, based on the spin-glasslike nature of the oxide layer. The occurrence of an exchange anisotropy effect between the ferromagnetic core and the surface results in a shift of the FC hysteresis loops below $50 \mathrm{~K}$, corresponding to the freezing temperature of the spin-glass phase.

It is worth noting that the possibility of observing the above described effects is strictly connected with obtaining systems modulated on a length scale in the nanometer regime. In fact, it has been proposed that the spin-glasslike state of the oxide surface is favored by the very reduced dimensions of its constituting crystallites and, moreover, it is clear that the exchange interaction would not be strong enough to affect considerably the magnetic behavior if the particle core and the oxide layer were not extended on a short and comparable scale length.

\section{ACKNOWLEDGMENTS}

The authors acknowledge Project Nos. CICYT MAT951042-C02-02 and PGC PB96-0863-C02-02. LDB acknowledges the European Commission for financial support (Grant No. ERBFMBI-CT95-0534).

${ }^{1}$ Y. Yoshizawa, S. Oguma, and K. Yamauchi, J. Appl. Phys. 64, 6044 (1988).

${ }^{2}$ E. F. Kneller and R. Hawig, IEEE Trans. Magn. MAG-27, 3588 (1991).

${ }^{3}$ S. S. Parkin, N. More, and K. P. Roche, Phys. Rev. Lett. 64, 2304 (1990).

${ }^{4}$ See for example, Magnetic Properties of Fine Particles, edited by J. L. Dormann and D. Fiorani (North-Holland, Amsterdam, 1992).

${ }^{5}$ W. Wagner, H. Van Swygenhoven, H. J. Höfler, and A. Wiedenmann, Nanostruct. Mater. 6, 929 (1995).

${ }^{6}$ W. Gong, H. Li, Z. Zhao, and J. Chen, J. Appl. Phys. 69, 5119 (1991).

${ }^{7}$ S. Gangopadhyay, G. C. Hadjipanayis, S. I. Shah, V. Papaefthymiou, and A. Kostikas, J. Appl. Phys. 70, 5888 (1991).

${ }^{8}$ J. Löffler, H. Van Swygenhoven, W. Wagner, J. Meier, B. Doudin, and J. $\mathrm{Ph}$. Ansermet, Nanostruct. Mater. 9, 523 (1997).

${ }^{9}$ H. Kisker, T. Gessmann, R. Würschum, H. Kronmüller, and H. E. Schaefer, Nanostruct. Mater. 6, 925 (1995).

${ }^{10}$ H. Gleiter, Prog. Mater. Sci. 33, 223 (1989).

${ }^{11}$ J. C. Sánchez-López and A. Fernández, Proceedings of Mechanically Alloyed, Metastable and Nanocrystalline Materials, ISMANAM-97, edited by M. D. Baró and S. Surinach (Trans. Tech. Publications Ltd, Ammersdorf, Switzerland, 1997), pp. 827-832.

${ }^{12}$ B. D. Cullity, in Introduction to Magnetic Materials (Addison-Wesley, London, 1972), p. 423.

${ }^{13}$ J. A. Mydosh, in Spin Glasses: An Experimental Introduction (Taylor and Francis, London, 1993), p. 91.

${ }^{14}$ S. Gangopadhyay, G. C. Hadjipanayis, B. Dale, C. M. Sorensen, and K. J. Klabunde, Nanostruct. Mater. 1, 77 (1992).

${ }^{15}$ K. Haneda and A. H. Morrish, Surf. Sci. 77, 584 (1978).

${ }^{16}$ S. Linderoth, S. Morup, and M. D. Bentzon, J. Mater. Sci. 30, 3142 (1995).

${ }^{17}$ R. H. Kodama, A. E. Berkowitz, E. J. McNiff, and S. Foner, J. Appl. Phys. 81, 5552 (1997).

${ }^{18}$ R. H. Kodama, S. A. Makhlouf, and A. E. Berkowitz, Phys. Rev. Lett. 79, 1393 (1997); K. Takano, R. H. Kodama, A. E. Berkowitz, W. Cao, and G. Thomas, ibid. 79, 1130 (1997).

${ }^{19}$ L. Del Bianco, A. Hernando, E. Bonetti, and E. Navarro, Phys. Rev. B 56, 8894 (1997). 